\title{
Simulation of synchrotron radiation from electron beams affected by vibrations and drifts
}

\author{
Sukho Kongtawong $\oplus^{*}$ \\ Department of Physics and Astronomy, Stony Brook University, New York, 11794-3800, USA \\ and NSLS-II, Brookhaven National Laboratory, Upton, New York, 11973, USA \\ Oleg Chubar ${ }^{\dagger}$ and Timur Shaftan \\ NSLS-II, Brookhaven National Laboratory, Upton, New York, 11973, USA
}

(Received 14 October 2021; accepted 24 January 2022; published 4 February 2022)

\begin{abstract}
This paper presents results of simulations of synchrotron radiation from electron beams affected by instabilities in position and angle at the source point. The goal of this work is to develop and test instrumentation for studies of the effects of the electron beam's vibrations and drifts on beamline performance at synchrotron light sources. To perform these simulations, numerical methods were developed and implemented in the Python version of the Synchrotron Radiation Workshop (SRW) code. The simulation results were compared against analytical formulas and experimental results obtained from an x-ray pinhole-camera diagnostics beamline at NSLS-II. We compared the measured data with the prediction by our code for amplitudes of orbit oscillation from 6-60 $\mu \mathrm{m}$ and frequencies between 1 and $40 \mathrm{~Hz}$, also taking into account the effects of misalignment of the pinhole. The simulation results are in good agreement with both analytical estimates and experimental data.
\end{abstract}

DOI: $10.1103 /$ PhysRevAccelBeams.25.024601

\section{INTRODUCTION}

Modern synchrotron light sources feature ultralow emittance and require highly stable electron beams to take full advantage of the high source brightness and coherence. The instability of the beam position/angle is typically required to be less than a few percent of the beam RMS size/angular divergence to avoid considerable negative impacts on the $\mathrm{x}$-ray beam properties. There are some studies on the effects of the instabilities and developments [1-4]. For complicated imaging beamlines, it would be difficult to understand the effects of the instabilities without performing simulations. Accurate simulations and benchmarking experiments can help to characterize and, possibly, control the negative impacts of the electron beam instabilities that otherwise can limit the performance of modern light source facilities. Recently, an optical instability calculation was implemented on a code based on a ray-tracing method [5]. This simulation allows a deeper understanding of the effects of optical instabilities and provides useful information for designers

\footnotetext{
*sukho.kongtawong@stonybrook.edu

chubar@bnl.gov

shaftan@bnl.gov
}

Published by the American Physical Society under the terms of the Creative Commons Attribution 4.0 International license. Further distribution of this work must maintain attribution to the author(s) and the published article's title, journal citation, and DOI. and engineers. Although, the ray-tracing method is a powerful implementation. Another calculation method based on the wave optics was also widely implemented for many applications which gives accurate results in the diffractiondominated regime. For example, calculating a pinhole cameras resolution or designing beamlines for modern light sources, such as coherent diffraction imaging or highresolution microscopy beamlines rely mainly on wave optic calculations [6-8]. In this work, we implemented the calculation of the electron beam instabilities into a code based on wave optics calculation.

To understand how the electron beam instability, such as the vibration of the beam in position and angle, affects beamlines, we need to calculate the synchrotron radiation, taking into account these instabilities of the source, and simulate the wavefront propagation through the x-ray optics of the beamlines. To accomplish this goal, we developed tools for analysis and simulations within the framework of the Synchrotron Radiation Workshop (SRW) code.

The SRW is a computer code that simulates synchrotron radiation (SR) and its propagation through beamline optics $[9,10]$. It is used for various applications, such as the design and optimization of insertion devices and beamline optical layouts for experiments and beam diagnostics $[11,12]$. The SR propagation through beamline optics is calculated using the Fourier optics approach.

In this paper, we present the results of the development of the SR emission and propagation calculation methods, including effects of the orbit instabilities of the electron 
beam at the source. The methods are implemented in the SRW's Python library [13]. The simulation methods that we developed will go a long way toward working out the specifics of beam stability at the source points as well as the requirements for achieving stability of the optical elements in long and complicated beamlines. With modern and future light sources offering high-brightness beams of synchrotron radiation, it is more important than ever to accurately predict the dilution of the sources beam quality at the detectors. Our tools allow us to simulate timedependent transients in the beam orbit positions and angles, for both random and regular models of the beam motion. It permits us to carefully assess the reduction of brightness and coherent flux on the sample, as well as the variation of the intensities, due to vibrations of the source. It will aid in setting specifications for the acceptable vibration levels of the accelerator and beamline components for present and future high-brightness light sources. It also paves the way for the development of simulations of optical elements vibrations, and the related effects in beamlines.

The results obtained using these methods were compared with an analytical formula and experimental data to verify the accuracy of the calculations. This work is a continuation of the beam stability studies at NSLS-II $[14,15]$; the results can be applied to other light source facilities as well.

\section{CALCULATION OF SYNCHROTRON RADIATION AND ITS PROPAGATION}

For a given magnetic field, the trajectory of a relativistic electron can be calculated by solving the differential equation of the Lorenz force with a set of initial conditions. From the trajectory (Fig. 1), SRW computes the electric field in the frequency domain $\mathbf{E}_{\omega}$ using the following equation [9]:

$\mathbf{E}_{\omega}=i e k \int_{-\infty}^{\infty}\left[\boldsymbol{\beta}-\hat{\mathbf{n}}\left(1+\frac{i}{k R}\right)\right] \frac{\exp (i k(c \tau+R))}{R} d \tau$,

where $e$ is the charge of the electron, $k=\omega / c$ is the wave number of the radiation, $\omega$ is the frequency of the SR, $c$ is the

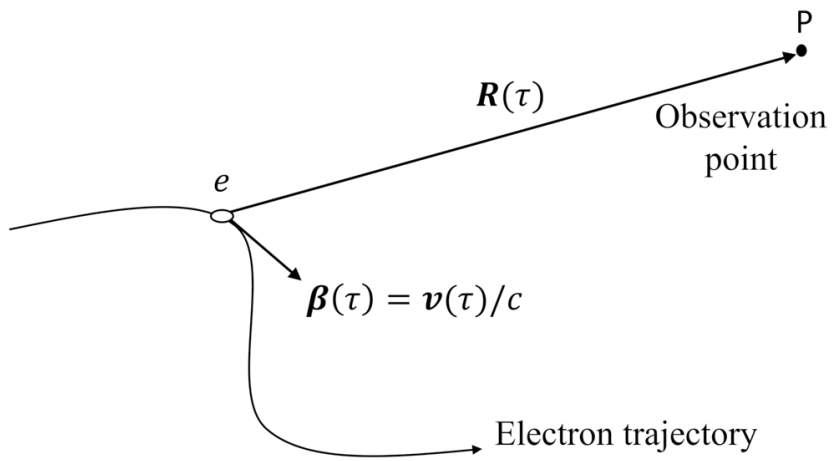

FIG. 1. A diagram showing the electron's trajectory and introducing variables for the SR calculation in Eq. (1).

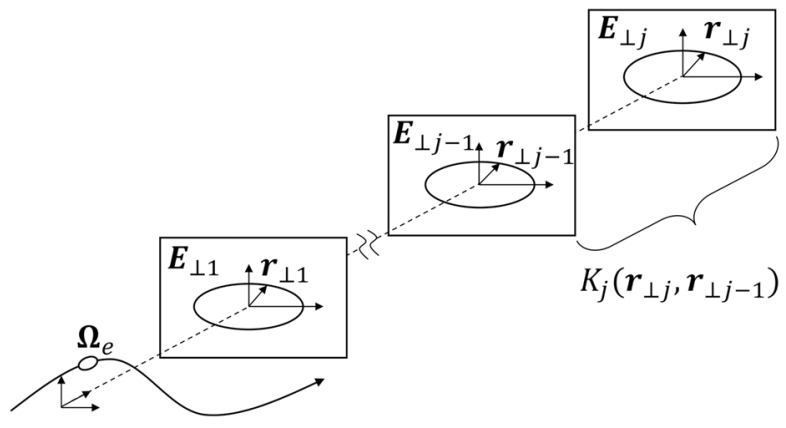

FIG. 2. A diagram introducing variables for the SR propagation in Eq. (2).

speed of light, $\boldsymbol{\beta}=\boldsymbol{\beta}(\tau)=\mathbf{v}(\tau) / c$ is the relative velocity of the electron, $R=R(\tau)=|\mathbf{R}(\tau)|$ is the instant distance between the source and the observation point, $\hat{\mathbf{n}}=\mathbf{R} / R$ is a unit vector directed from the source to the observation point, and $i$ is the imaginary number. This equation is an exact formula for the SR calculation, so it is valid for both near-field and far-field approximations.

For the SR propagation, the code implements the Fourier optics and compatible methods [16,17]. Within this framework, the transverse components of the frequency-domain electric field at a $j$ th optical element $\mathbf{E}_{\perp j}$ can be calculated from the transverse components of the electric field at a $(j-1)$ th optical element $\mathbf{E}_{\perp j-1}$, which is also determined by the initial conditions of the electron (Fig. 2). The calculation is done using a propagator:

$$
\begin{aligned}
\mathbf{E}_{\perp j}\left(\mathbf{r}_{\perp j}, \boldsymbol{\Omega}_{\mathrm{e}}, \omega\right)= & \iint K_{j}\left(\mathbf{r}_{\perp j}, \mathbf{r}_{\perp j-1}, \omega\right) \\
& \times \mathbf{E}_{\perp j-1}\left(\mathbf{r}_{\perp j-1}, \mathbf{\Omega}_{\mathrm{e}}, \omega\right) d x_{j-1} d y_{j-1},
\end{aligned}
$$

where $K_{j}\left(\mathbf{r}_{\perp j}, \mathbf{r}_{\perp j-1}\right)$ is a kernel associated with a $j$ th optical element (including drift spaces), $\mathbf{r}_{\perp j}=\mathbf{r}_{\perp j}\left(x_{j}, y_{j}\right)$ is a transverse vector of a point on the plane where $\mathbf{E}_{\perp j}$ is considered, and $\boldsymbol{\Omega}_{\mathrm{e}}=\left(x_{\mathrm{e}}, y_{\mathrm{e}}, x_{\mathrm{e}}^{\prime}, y_{\mathrm{e}}^{\prime}, \gamma_{\mathrm{e}}, z_{\mathrm{e}}\right)$ is the initial coordinates of the electron in 6D phase space, where $x_{\mathrm{e}}$, $y_{\mathrm{e}}$ are the initial horizontal and vertical position of the electron, respectively, $x_{\mathrm{e}}^{\prime}, y_{\mathrm{e}}^{\prime}$ are the initial angles, $\gamma_{\mathrm{e}}$ is the electron's relative energy, and $z_{\mathrm{e}}$ is the initial longitudinal position. Here, $\mathbf{E}_{\perp j}\left(\mathbf{r}_{\perp j}, \boldsymbol{\Omega}_{\mathrm{e}}, \omega\right)$ depends on variable $\mathbf{r}_{\perp j}$, the transverse coordinate of the observation plane, while $\boldsymbol{\Omega}_{\mathrm{e}}$ and $\omega$ serve as parameters describing the coordinate of the electron and SR frequency. An example

is a kernel $K=-i k L \exp (i k S) /\left(2 \pi S^{2}\right)$, where $S=$ $\sqrt{L^{2}+\left(x_{j}-x_{j-1}\right)^{2}+\left(y_{j}-y_{j-1}\right)^{2}}$ and $L$ is the longitudinal distance between the $j$ th and $(j-1)$ th planes. Then Eq. (2) turns into the well-known Huygens-Fresnel principle.

The calculation presented by Eqs. (1) and (2) applies to the case of a single electron traversing a magnet. To calculate SR 
in the case of a beam with a finite emittance, we need to take into account the contributions from many electrons with different initial conditions forming the beam. The code has a function to calculate the propagation of partially coherent wavefronts using the following equation [17]:

$$
I_{j}\left(\mathbf{r}_{\perp j}, \omega\right)=\int\left|\mathbf{E}_{\perp j}\left(\mathbf{r}_{\perp j}, \boldsymbol{\Omega}_{\mathrm{e}}, \omega\right)\right|^{2} f\left(\boldsymbol{\Omega}_{\mathrm{e}}\right) d \boldsymbol{\Omega}_{\mathrm{e}},
$$

where $I_{j}$ is the intensity contributed from all the electrons in the beam, $\mathbf{E}_{\perp j}$ is the transverse electric field from Eq. (2), $f\left(\boldsymbol{\Omega}_{\mathrm{e}}\right)$ is the normalized particle density distribution in $6 \mathrm{D}$ phase space, and $d \Omega_{\mathrm{e}}=d x_{\mathrm{e}} d y_{\mathrm{e}} d x_{\mathrm{e}}^{\prime} d y_{\mathrm{e}}^{\prime} d \gamma_{\mathrm{e}} d z_{\mathrm{e}}$ is the infinitesimal phase space volume of the beam.

Equation (3) is implemented by summing up contributions from many electrons with randomized initial conditions based on the beam parameters, i.e., beam size, angular divergence, and energy spread. Note that if the system is spatially invariant, Eq. (3) reduces to a convolution with respect to a linear combination of the positions and angles.

Some beamlines require calculations for polychromatic $\mathrm{x}$-rays over a large spectral range. The transmitted radiation spectrum depends on the optical elements of the system, such as filters. The total intensity of the polychromatic radiation $I_{j}\left(\mathbf{r}_{\perp j}\right)$ can be calculated by

$$
I_{j}\left(\mathbf{r}_{\perp j}\right)=\int I_{j}\left(\mathbf{r}_{\perp j}, \omega\right) g(\omega) d \omega,
$$

where $g(\omega)$ is a spectral attenuation coefficient associated with the optical elements of the system.

Combining Eqs. (1)-(4) with the knowledge of a kernel for each optical element, we can calculate the electric field at any location along a beamline. The initial conditions, e.g., initial positions and angles of electrons, can be adjusted to simulate misalignment effects in electron trajectories. However, studying the effects of beam instability requires a time-dependent variation of these initial parameters $\boldsymbol{\Omega}_{\mathrm{e}}$. The SR calculation, including the effects of the time-dependent variation, will be discussed in the following sections.

\section{INCLUDING ELECTRON ORBIT INSTABILITIES INTO THE SIMULATION}

We modified the SRW Python library by adding contributions of noise to the initial conditions of the electron beam. The beam vibration can be added directly to the beam initial conditions since the frequency range of the vibrations is much smaller than that of the SR fields. The initial conditions can be modified by adding a timedependent noise component:

$$
\boldsymbol{\Omega}_{\mathrm{e}}(t)=\boldsymbol{\Omega}_{\mathrm{b}}+\boldsymbol{\Omega}_{\mathrm{n}}(t),
$$

where $\boldsymbol{\Omega}_{\mathrm{b}}$ is the contribution associated with beam parameters, i.e., beam size, angular divergence, and energy spread, and $\boldsymbol{\Omega}_{\mathrm{n}}(t)$ is the contribution from the timedependent noise.

During a simulation, random values of the initial parameters $\boldsymbol{\Omega}_{\mathrm{b}}$ are generated assuming Gaussian distributions for the beam size, divergence, and energy spread, and used as initial conditions to simulate SR from individual electrons. The time-series data of the noise parameters $\boldsymbol{\Omega}_{\mathrm{n}}(t)$ can be imported from the real measurements or generated in Python. For example, we can generate a timeseries of a simple harmonic function of the electron position in the source point:

$$
y_{\mathrm{n}}(t)=a \sin \left(2 \pi f t+\phi_{0}\right),
$$

where $f$ is the frequency of the oscillation, $\phi_{0}$ is the initial phase, and $t$ is the time. We constrain the observation time by the interval of the data collection of the detector at the beamline, i.e., the time ranges from 0 to $T$, in which $T$ corresponds to the data collection. The values of $\boldsymbol{\Omega}_{\mathrm{b}}$ and $\boldsymbol{\Omega}_{\mathrm{n}}$ are then used as electron initial conditions, following Eq. (5).

Assuming that noises can be considered as stationary stochastic processes [18] within $T$, we can use random numbers, with associated stationary distributions, for the simulations of noise. The code that we developed provides random numbers obeying various distributions for $\boldsymbol{\Omega}_{\mathrm{n}}$, such as a uniform random distribution, a Gaussian distribution, or an arbitrary function of uniform random numbers. The noise can be independently assigned to any of the parameters in $\boldsymbol{\Omega}_{\mathrm{n}}=\left(x_{\mathrm{n}}, y_{\mathrm{n}}, x_{\mathrm{n}}^{\prime}, y_{\mathrm{n}}^{\prime}, \gamma_{\mathrm{n}}, z_{\mathrm{n}}\right)$. All variables of the initial conditions, such as positions and angles, can be assigned using arbitrary time-dependent functions simultaneously.

This method is suitable for vibrations that are slow enough such that the intensity variations can be resolved with the detector of finite time resolution. For high frequency vibrations where the period of oscillation is much smaller than the characteristic time of the detector, this method requires a large number of initial conditions in order to achieve accurate results, which might not be efficient. In this high-frequency regime, however, the detector will see the beam with a larger emittance [19]. Hence, we can implement the calculation by using a larger beam emittance in $\boldsymbol{\Omega}_{\mathrm{b}}$ instead of using a time-dependent variation with high-frequency vibrations in $\boldsymbol{\Omega}_{\mathrm{n}}(t)$.

In the next section, the average intensity is compared with analytical estimates to test our calculation methods. For the analytical estimates, the average intensity can be calculated by

$$
\bar{I}\left(\mathbf{r}_{\perp}\right)=\frac{1}{T} \int_{0}^{T} I\left(\mathbf{r}_{\perp}, \boldsymbol{\Omega}_{\mathrm{e}}(t)\right) d t .
$$


Using Eq. (7), we can analytically calculate the average intensity of a well-known case of SR and compare it with the intensity calculated from the simulation.

\section{COMPARISON WITH ANALYTICAL ESTIMATES}

As an example, we simulated a simple case of SR from a constant magnetic (dipole) field in projection geometry, without any beamline optics. The noise was introduced to the vertical angle of the electron beam entering the dipole. Figure 3 shows the intensity distributions with and without the noise at a distance of $5 \mathrm{~m}$ from the source. The photon energy is $2.39 \mathrm{keV}$, which corresponds to the critical energy of SR emitted by $3 \mathrm{GeV}$ electrons in a $0.4 \mathrm{~T}$ magnetic field of an NSLS-II dipole magnet.

We can compare these results with an analytical formula for this case. The far-field radiation can be calculated using an equation (Gaussian unit) [20]:

$$
\begin{aligned}
\left(\frac{d^{2} I}{d \omega d \Omega}\right)_{\mathrm{G}}= & \frac{e^{2}}{3 \pi^{2} c}\left(\frac{\omega \rho}{c}\right)^{2}\left(\frac{1}{\gamma^{2}}+\theta^{2}\right)^{2} \\
& \times\left[K_{2 / 3}^{2}(\xi)+\frac{\theta^{2}}{1 / \gamma^{2}+\theta^{2}} K_{1 / 3}^{2}(\xi)\right],
\end{aligned}
$$
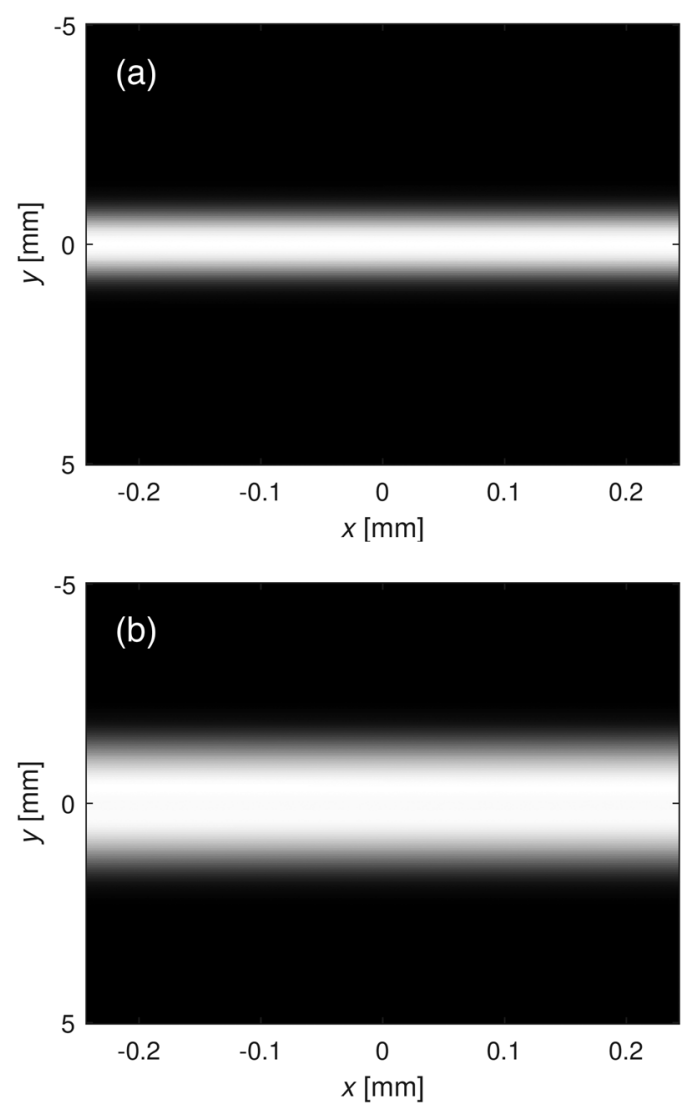

FIG. 3. Simulated intensity of SR (at $2.39 \mathrm{keV}$, critical energy) from a filament electron beam for (a) no noise and (b) a harmonic noise with amplitude $a=0.2 \mathrm{mrad}$. where $\xi=\frac{\omega \rho}{3 c}\left(\frac{1}{\gamma^{2}}+\theta^{2}\right)^{3 / 2}, \rho$ is the radius of the electron's trajectory, $\gamma$ is the Lorenz factor related to the electron's energy, $\theta$ is the latitude (vertical) angle from the particle to the observation point, and $K_{\alpha}(\alpha=1 / 3,2 / 3)$ are the modified Bessel functions of the second kind.

We can consider $\theta$ in Eq. (8) as the variable affected by the noise in the following way:

$$
\theta=\frac{y-y_{\mathrm{n}}(t)}{R}-y_{\mathrm{n}}^{\prime}(t)
$$

where $y$ is the vertical position on the plane of observation, $y_{\mathrm{n}}(t)$ is the noise for the vertical electron beam position, $y_{\mathrm{n}}^{\prime}(t)$ is the noise for the vertical electron beam angle, and $R$
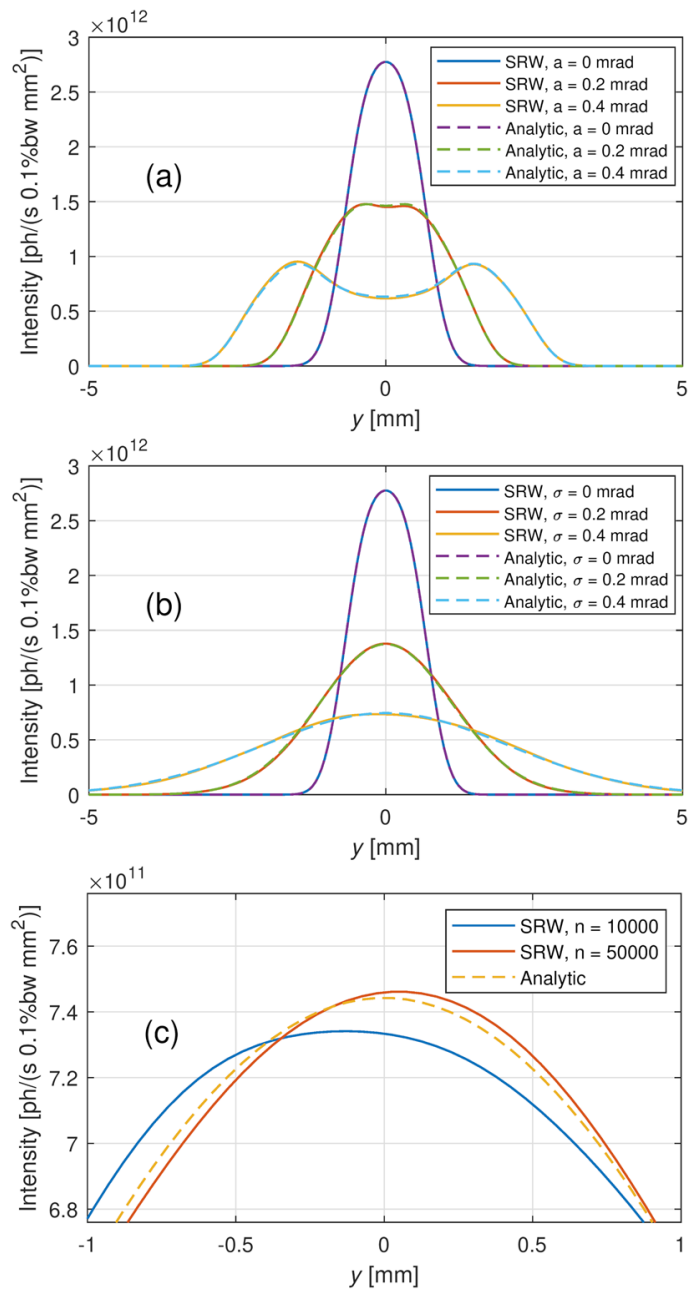

FIG. 4. Simulation and analytical results for vertical cuts of the average intensity distributions at $2.39 \mathrm{keV}$ photon energy for (a) a harmonic noise in the vertical angle (one period of oscillation) with amplitudes of 0,0.2,0.4 mrad; (b) a Gaussian noise with the standard deviations of 0,0.2,0.4 mrad; and (c) comparison of the simulation results from 10,000 and 50,000 initial conditions with the analytical result of the Gaussian noise with $\sigma=0.4 \mathrm{mrad}$. The magnetic field and beam parameters are the same as those used in the calculations illustrated in Fig. 3. 
is the distance between the source and the observation plane. By combining Eqs. (8) and (9), we can calculate the intensity at any point on the observation plane. Note that for a large distance $R$, the system is more sensitive to the angle of the beam than the position.

Figure 4 shows comparisons of the intensity distribution as a function of the vertical position between the SRW simulation and the analytical calculation for a harmonic and a Gaussian noise at different amplitudes. The time-average intensity for the analytical estimates was calculated numerically using Eq. (7). The intensity is integrated over large time interval $T$ for the Gaussian noise case and $T$ of one period of oscillation for the harmonic noise case; for example, if $f=20 \mathrm{~Hz}$, then $T=50 \mathrm{~ms}$ [from Eq. (6)].

It can be seen from Fig. 4 that the results from the simulation are in good agreement with the analytical solution. In this example, the numerical simulations were done for 10,000 electrons' initial conditions. We can see small errors in the intensity of less than $2 \%$, which are attributed to the chosen number of the initial conditions. Somewhat larger errors in the intensity distribution, corresponding to larger amplitude cases, are likely because of the much lower particle density distribution in the case of larger amplitudes. Increasing the number of the initial conditions can reduce the errors as shown in Fig. 4(c).

\section{BENCHMARKING SIMULATION AGAINST BEAM MEASUREMENT AT THE NSLS-II X-RAY PINHOLE CAMERA BEAMLINE}

This section details how the simulation method described above was used to interpret the results of an actual experiment performed at the x-ray pinhole-camera diagnostics beamline that was developed for measuring parameters of the electron beam in the NSLS-II storage ring [21], which was developed for measuring parameters of the electron beam in the NSLS-II storage ring. The system consists of an $80 \times 25 \mu \mathrm{m}$ (horizontal $\times$ vertical) rectangular pinhole located at a distance of $3.033 \mathrm{~m}$ from the electron beam source point in a bending magnet (Fig. 5). A scintillator, converting x-rays to visible light, is located $13.095 \mathrm{~m}$ after the pinhole. The electron beam image in this

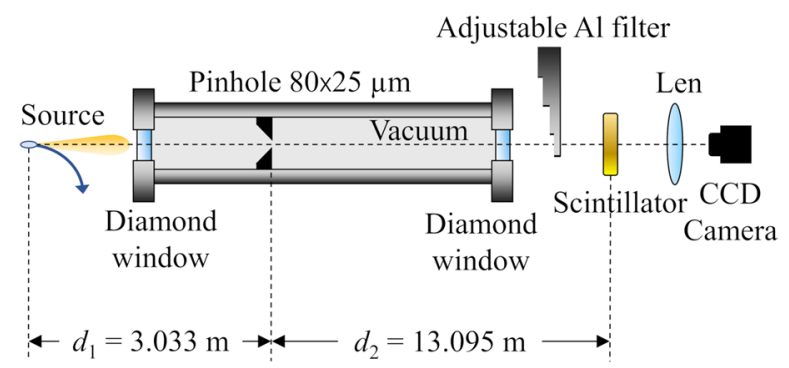

FIG. 5. A simplified diagram of the x-ray pinhole-camera diagnostic beamline at NSLS-II. beamline is formed by $\mathrm{x}$-rays within a large spectral bandwidth ranging from 1 to $40 \mathrm{keV}$.

To estimate the spatial resolution of the pinhole camera, we calculated its point spread function (PSF), i.e., the x-ray intensity distribution at the position of the scintillator, created by a filament (zero-emittance) electron beam. The calculations were done first for a number of fixed photon energies, covering the spectrum of bending magnet SR and taking into account spectral attenuation of the entire beamline and spectral efficiency of x-ray conversion by the scintillator. In the calculations we used the wave-optics methods implemented in the SRW code. After this, the individual monochromatic intensity distributions were integrated [see Eq. (4)] to simulate the polychromatic intensity distribution that can be treated as the PSF of the pinhole camera. The results of these calculations are presented in Fig. 6(a). At low photon energies $(<5 \mathrm{keV})$, the size of the obtained intensity distributions is large due to the effects of diffraction, whereas at high energies, e.g., $30 \mathrm{keV}$, the monochromatic PSF indicates the modulation related to near-field diffraction. We can see that the size of the PSF at $10 \mathrm{keV}$ is close to the size of the polychromatic PSF. Figure 6(b) shows vertical intensity profiles from 2D convolutions between the PSF and the electron beam distribution. It can also be seen that the monochromatic intensity at $10 \mathrm{keV}$ matches well the polychromatic x-ray
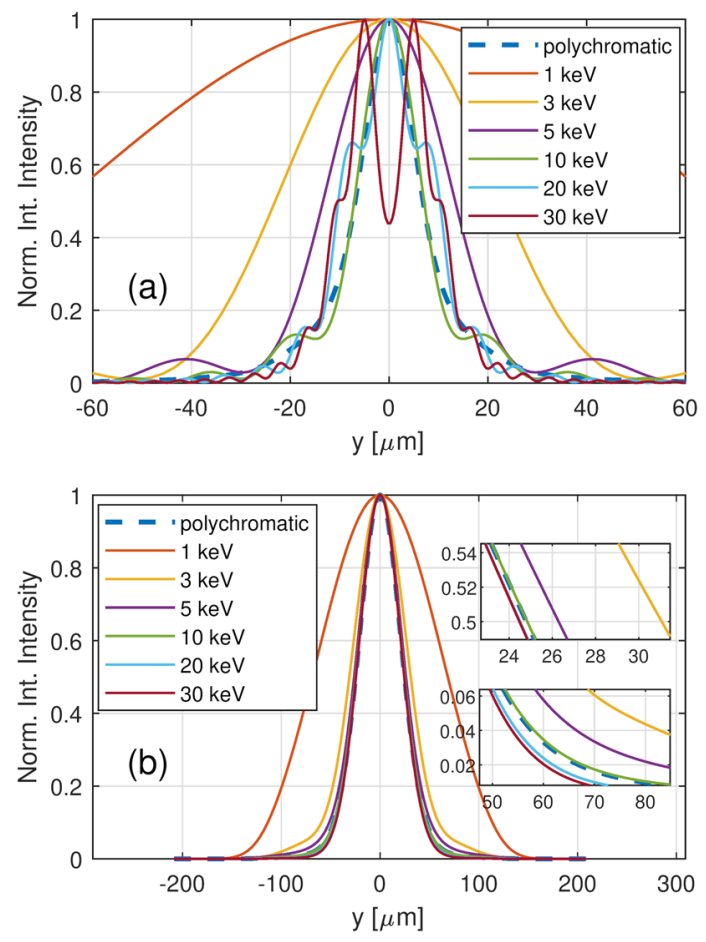

FIG. 6. Simulated normalized intensity (vertical profiles) from the $\mathrm{x}$-ray pinhole-camera diagnostic beamline for polychromatic $\mathrm{X}$-rays (dashed lines) and monochromatic X-rays (solid lines) for (a) the PSF and (b) the convolution of the PSF with the particle density distribution in the electron beam. 

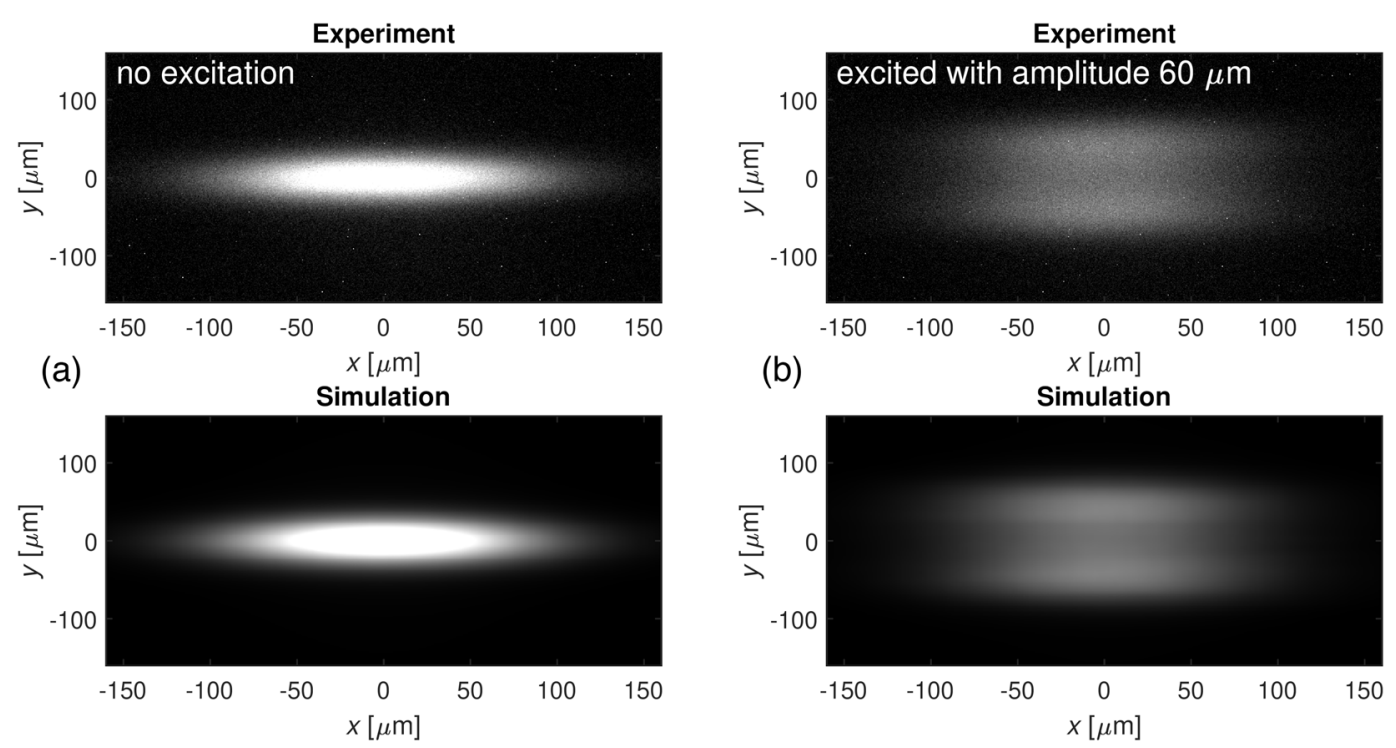

FIG. 7. Images of the pinhole camera from the experiment (top) and simulation (bottom) for (a) no excitation and (b) an excitation with an amplitude of $60 \mu \mathrm{m}$, while the beam size was about $20 \mu \mathrm{m}$.

intensity profile. From these estimations, we chose the energy of $10 \mathrm{keV}$ for the simulations described in the following sections (since the monochromatic partially coherent calculations are much more CPU-efficient than the polychromatic simulations).

To compare the experimental results and the simulations, we excited the electron beam orbit in the vertical plane with harmonic signals using a fast corrector. The excitation was done with different amplitudes and frequencies. Figure 7(a) shows the image plots from the experiment (top) and the simulation (bottom) under the conditions of no excitation. Figure 7(b) shows the image obtained when we excited the beam with a large amplitude of $60 \mu \mathrm{m}$ at $20 \mathrm{~Hz}$. The exposure time of the CCD camera was set to $T=50 \mathrm{~ms}$ for all of the following results. The number of oscillation periods used in the simulations shown in Fig. 7(b), according to Eq. (6), was

$$
N=f T=(20 \mathrm{~Hz})(50 \mathrm{~ms})=1 .
$$

Note that, in the experiment, the beam size at the source was about $160 \times 20 \mu \mathrm{m}$ (horizontal $\times$ vertical). The beam current was $40 \mathrm{~mA}$. Also note that the images shown here and in the following sections were rescaled to reproduce images at the source, i.e., were divided by the image magnification of the optical system.

Figure 8 shows the comparison between the intensity distributions for various amplitudes at $20 \mathrm{~Hz}(N=1)$. The plots show integrated intensity in the vertical plane. While the simulation can calculate the intensity in absolute units, the experimental data were collected in arbitrary units. We calibrated the intensity from the simulations such that it would match the experimental data and normalized them with the intensity from the no-excitation case [Fig. 8(a)]. For all other conditions, we used the same calibration values. Figure 8(b) shows the image profile with an excitation of $6 \mu \mathrm{m}$, which is about $30 \%$ of the beam size. There was not much difference compared to the data without an excitation in Fig. 8(a). We note that the maximum intensity dropped by about $3 \%$ and the beam size increased by less than $3 \%$. To see a clear behavior of the effects of the noise, we increased the amplitude of the excitation up to $60 \mu \mathrm{m}$ (three times the beam size). Figure 8(c) shows the image corresponding to the amplitude of $30 \mu \mathrm{m}$. We can see that the shape distorts from the Gaussian function due to the harmonic noise starting to dominate the beam size. Figure 8(d) shows the noise of $60 \mu \mathrm{m}$ amplitude, where we can see two peaks on the image profile created by a harmonic distribution similar to that indicated in Fig. 4(a). Note that the blue lines on each plot in Fig. 8 show many data sets from different measurements. However, since $N$ was an integer number in this case, the images were steady, so we see all the lines close to each other.

The next comparison shows the effects of low-frequency noise or slow-drifts. We excited the beam with harmonic signals at $1 \mathrm{~Hz}(N=0.05)$. We compared the results with amplitudes of excitation of $6 \mu \mathrm{m}$ and $60 \mu \mathrm{m}$ [Figs. 9(a)-9(b)]. In this case, we can see the images have moved along the horizontal axis, corresponding to different initial phases of oscillations. The results from the simulation are in good agreement with the experiment. We can also see that the intensity was slightly reduced when the beam moved away from the center.

We then compared the effects of the noise for various frequencies while keeping the amplitude at $60 \mu \mathrm{m}$. Figures 9(c)-9(d) show the plots for frequencies of $5 \mathrm{~Hz}$ 

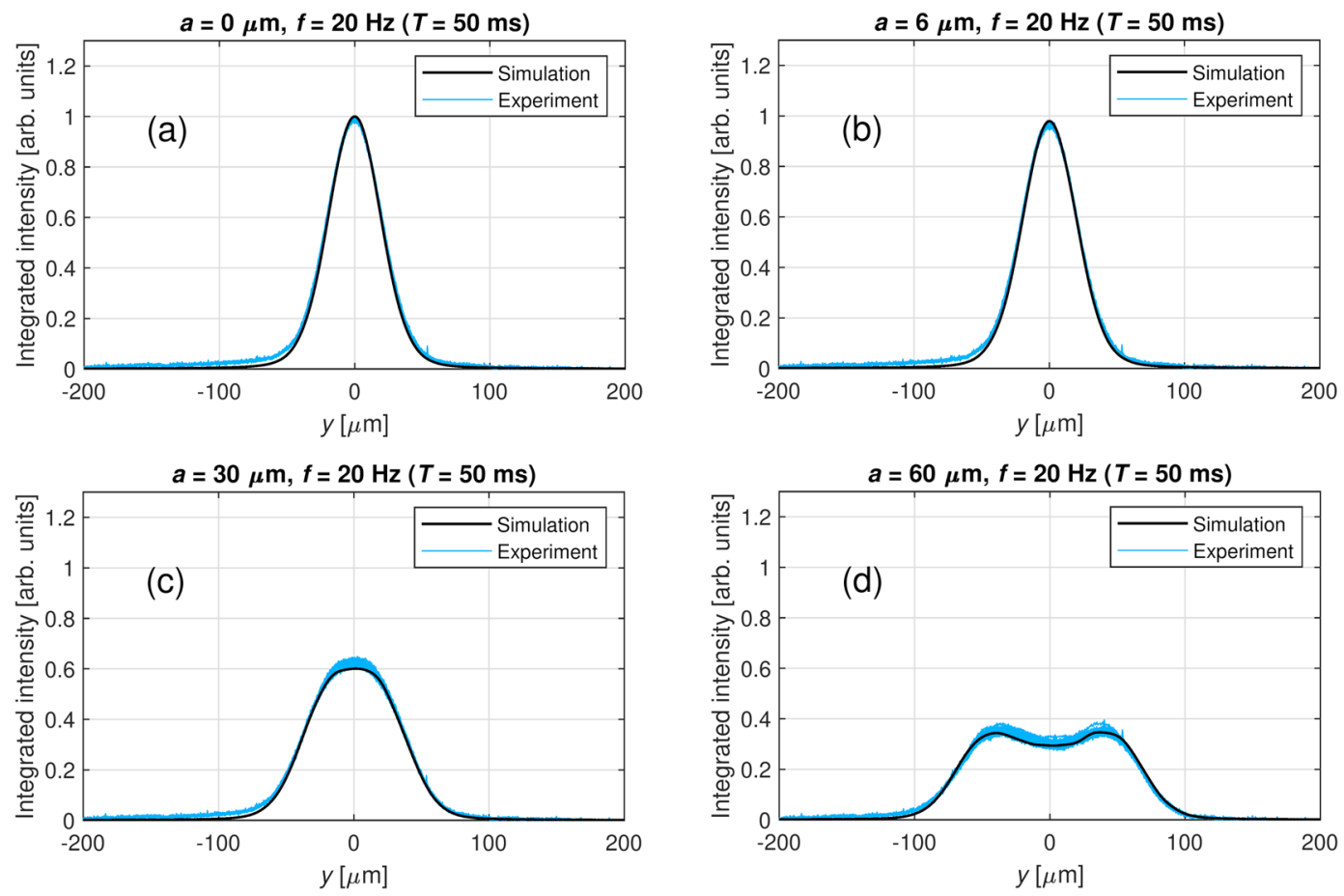

FIG. 8. Integrated intensity (vertical profiles) from the experiment (blue lines) and simulation (black lines) with (a) no excitation and excited with amplitudes (b) $6 \mu \mathrm{m}$, (c) $30 \mu \mathrm{m}$, and (d) $60 \mu \mathrm{m}$.

and $30 \mathrm{~Hz}$ (corresponding to $N=0.25$ and 1.5, respectively). Similar to the $1 \mathrm{~Hz}$ case, the values of $N$ are not integer numbers; due to this, the images moved and we can see many different lines. These results show more complicated behavior, but nevertheless, they have some details in common. The intensity was higher when the beam was at the turning point of the oscillation. Also, the image sizes tended to be smaller on the edge than when
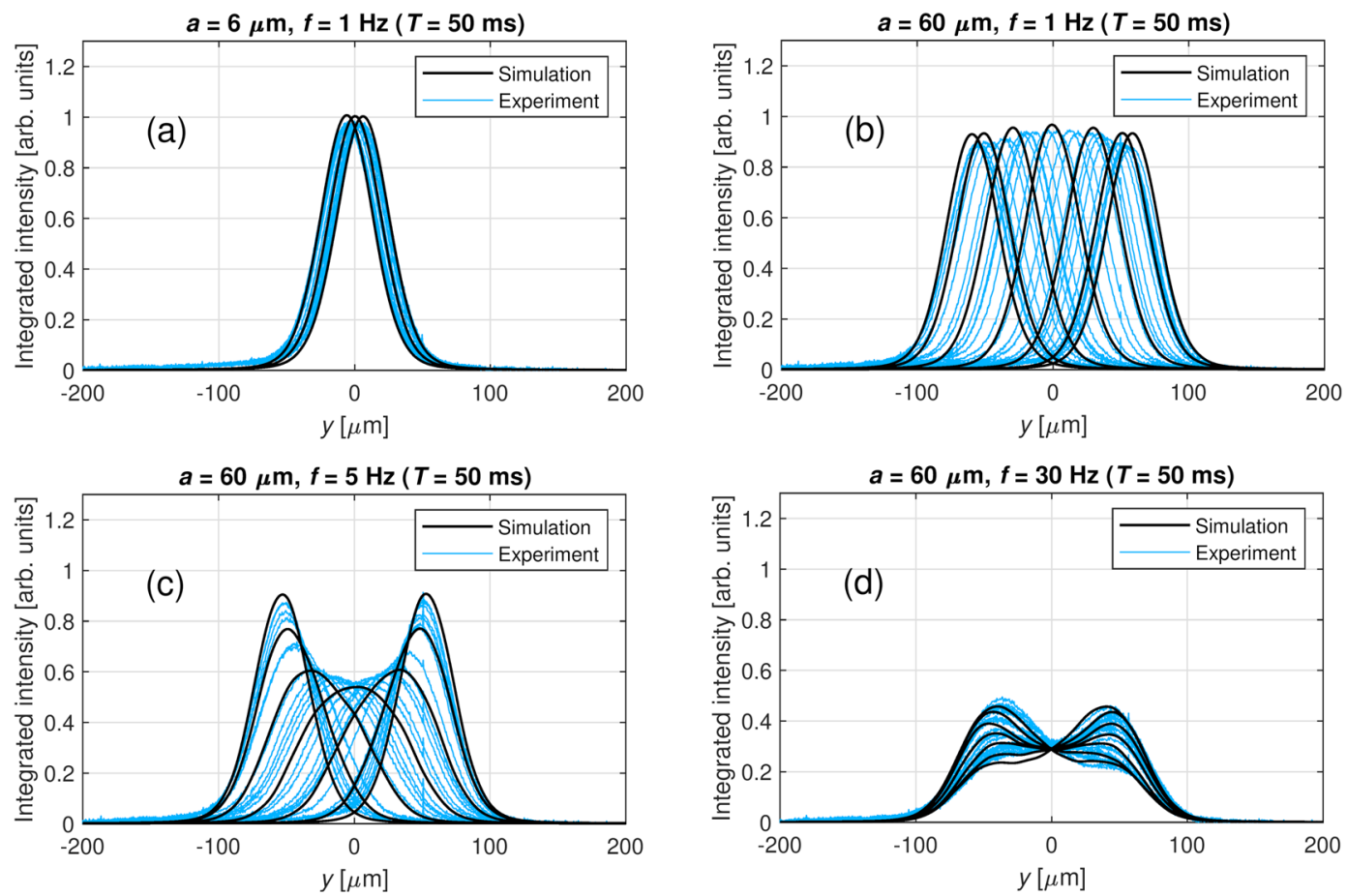

FIG. 9. Integrated intensity (vertical profiles) from the experiment (blue lines) and simulation (black lines). The beam was excited with amplitudes and frequencies of (a) $6 \mu \mathrm{m}, 1 \mathrm{~Hz}$, (b) $60 \mu \mathrm{m}, 1 \mathrm{~Hz}$, (c) $60 \mu \mathrm{m}, 5 \mathrm{~Hz}$, and (d) $60 \mu \mathrm{m}, 30 \mathrm{~Hz}$. 

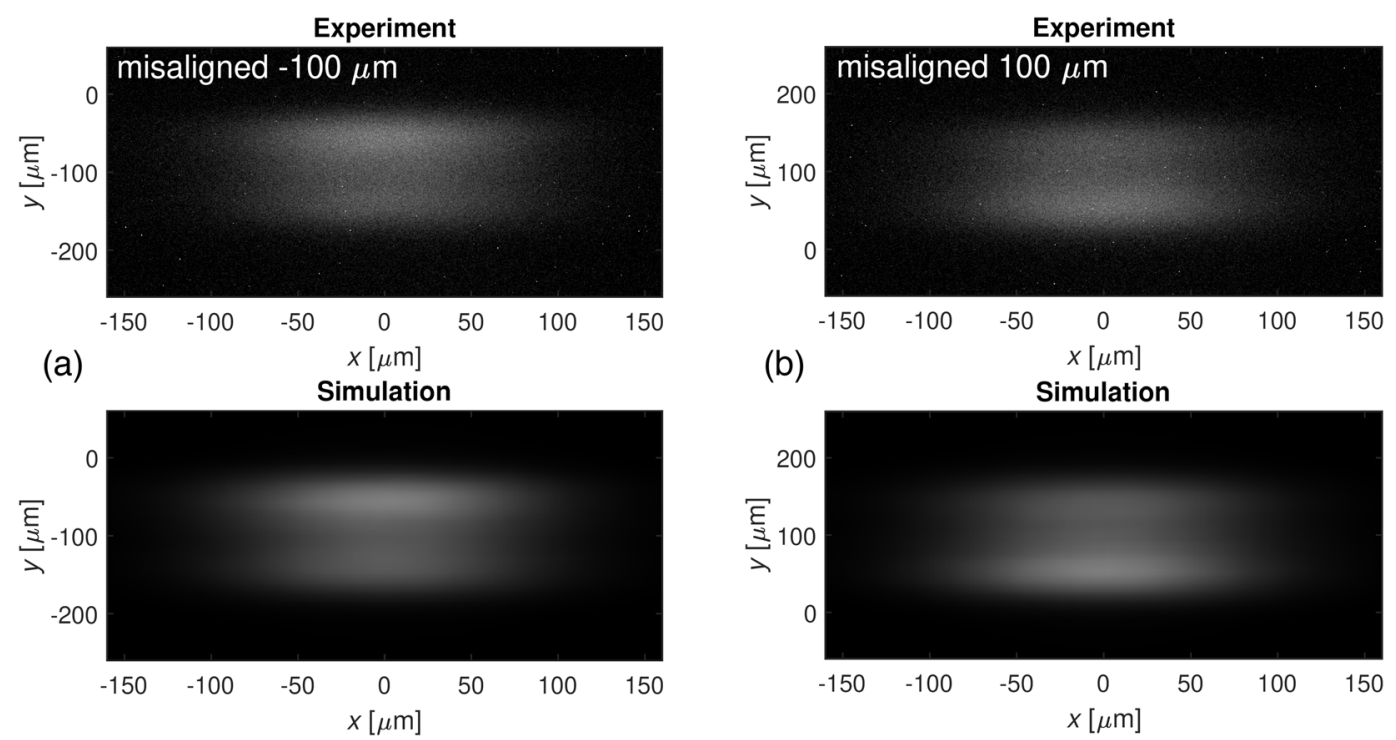

FIG. 10. Images of the pinhole camera from the experiment (top) and the simulation (bottom) with a vertical pinhole misalignment of (a) $-100 \mu \mathrm{m}$ and (b) $100 \mu \mathrm{m}$. The beam was excited with an amplitude of $60 \mu \mathrm{m}$.

the beam was at the center of the oscillation. This effect was expected since, under the conditions of the harmonic noise, the beam moved more slowly and stayed longer around the turning point compared to when it was at the center of the oscillation. Hence, after averaging, the intensity around the edge is higher, which is in contrast to a slow-drift case when the intensity around the edge is slightly lower than at the center [see Figs. 9(a)-9(b)]. For very slow frequency (and stable beam) cases, the effect of misalignment dominates the effect of the beam motion, which will be discussed later in this section. The simulation results are in good agreement with the experiment.

Finally, we present the effects of the misalignment between the pinhole and the electron beam. We collected the data with different relative positions between the center of the pinhole and the beam position. Figure 10 shows
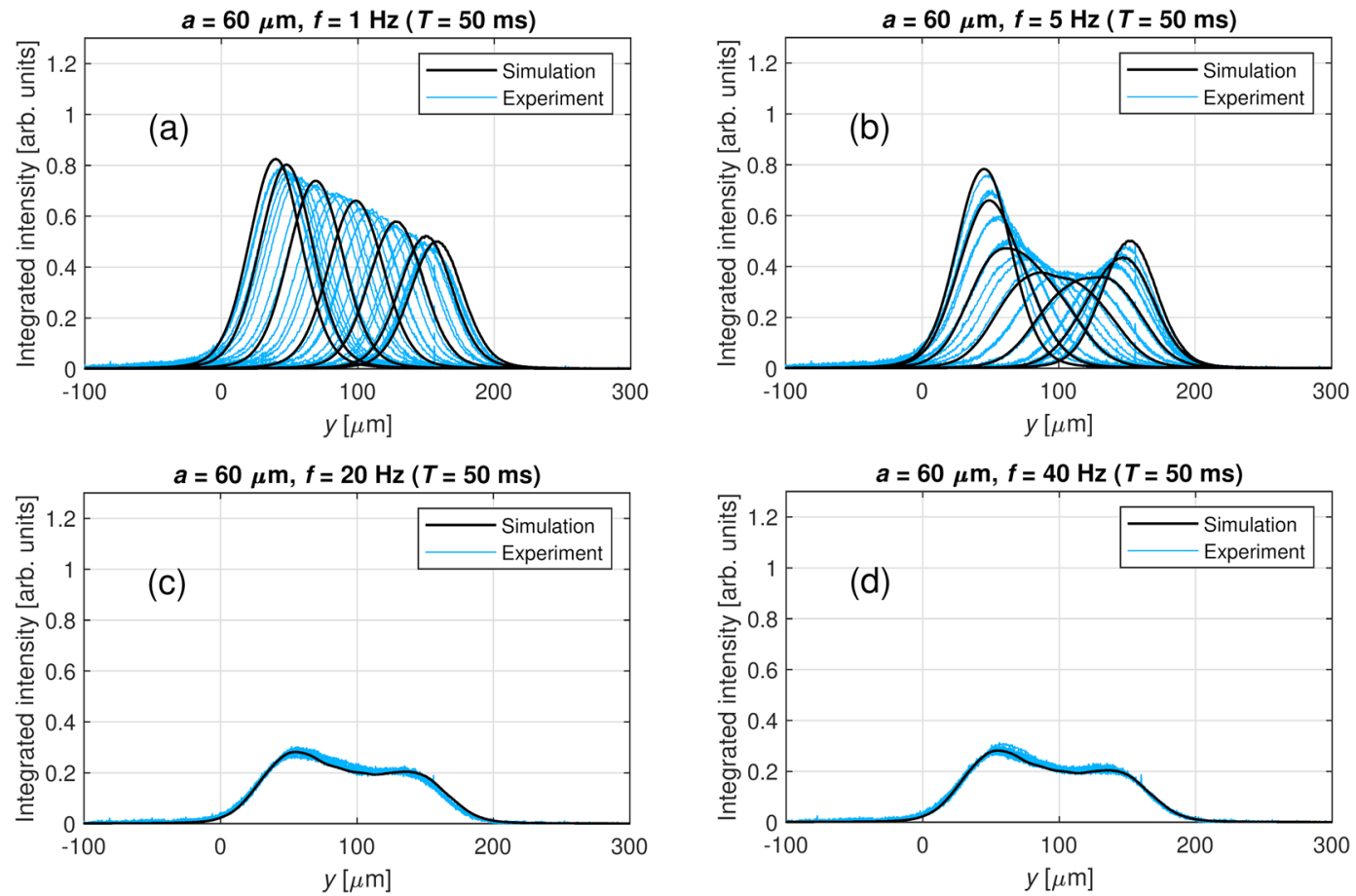

FIG. 11. Integrated intensity (vertical) with a pinhole misalignment of $100 \mu \mathrm{m}$ from the experiment (blue lines) and simulation (black lines). The beam was excited with an amplitude of $60 \mu \mathrm{m}$ and frequencies of (a) $1 \mathrm{~Hz}$, (b) $5 \mathrm{~Hz}$, (c) $20 \mathrm{~Hz}$, and (d) $40 \mathrm{~Hz}$. 
images from the experiment and simulation with the pinhole misaligned by $-100 \mu \mathrm{m}$ and $100 \mu \mathrm{m}$. The beam was excited with an amplitude of $60 \mu \mathrm{m}$. We can see that the images change between different misalignments. This indicates that the pinhole camera system is not spatially invariant. The relative position of the beam and the pinhole affects the image profiles on the screen.

Figure 11 shows the integrated intensity with a pinhole misalignment of $100 \mu \mathrm{m}$ for different frequencies. The overall behavior is the same as what we see in Figs. 8-9, except that the intensity profiles are somewhat asymmetric. At a very low frequency [Fig. 11(a)], the images moved slowly and the maximum intensity of each image reduced quite linearly as a function of the position. For higher frequencies, we can see two peaks from the effect of the beam motion as before. The images were not steady for noninteger values of $N$ [Figs. 11(a)-11(b)] and were steady for the integer values [Figs. 11(c)-11(d)]. The simulation results match the experimental data.

This section presents a comparison between the simulation and experiment with the x-ray pinhole-camera diagnostic beamline. We excited the beam from low to high amplitudes to study the effects of the noise. The purpose was to verify the accuracy of the simulation in different conditions. In reality, the baseline noises in synchrotron light sources are usually suppressed to be less than $10 \%$ of the beam size. However, some beamlines require higher beam stability and are affected by the noise in the real operation. The ultimate goal of this work is to apply the simulation to study the impact of the noise on more sensitive user beamlines at NSLS-II.

\section{SUMMARY AND DISCUSSION}

We developed methods to calculate the synchrotron radiation from electron beams affected by noise. The simulation was developed and implemented in the SRW's Python library. The developed functions can generate several noise distributions, such as uniform random numbers and Gaussian distributions, as well as accept userdefined arbitrary functions. The simulation can also import raw data from real measurements.

We verified the results from numerical simulations with analytical calculations and experimental data for various conditions. The first comparison focused on checking the simulation results with an analytical solution. We used a simple case of (far-field) SR from a constant magnetic field to benchmark the data. We compared the results with a harmonic noise and a Gaussian noise. Both cases were found to be in good agreement with the analytical solution-within $2 \%$ for 10,000 electrons' initial conditions of simulation. If we need higher accuracy, we can run the simulation with a larger number of initial conditions. Second, we compared the simulation results with the experimental data from the x-ray pinhole-camera diagnostic beamline at NSLS-II. We compared the results for various conditions, such as different amplitudes and frequencies. The effects of a pinhole misalignment were also discussed. The results from the simulation are in good agreement with the experiment within the uncertainties of the experimental data.

This work is a continuation of studies on electron and x-ray beam stability at NSLS-II. The goal of this development is to apply our simulation tools to beamlines that are most sensitive to and, therefore, are most affected by electron beam instability. It can be also applied to any beamline in general.

\section{ACKNOWLEDGMENTS}

This research used resources of the National Synchrotron Light Source II, a U.S. Department of Energy (DOE) Office of Science User Facility operated for the DOE Office of Science by Brookhaven National Laboratory under Contract No. DE-SC0012704.

[1] R. O. Hettel, Rev. Sci. Instrum. 73, 1396 (2002).

[2] A. Lumpkin, B. Yang, C. Yao, and L. Emery, in Proceedings of the 2003 Particle Accelerator Conference (IEEE, Portland, 2003), Vol. 4, pp. 2423-2425, https://ieeexplore .ieee.org/document/1289141.

[3] C. Steier, in 1st BES Light Sources Beam Stability Workshop (The Advanced Photon Source, 2018).

[4] G. M. Wang, Presented at IPAC'21 in Campinas, SP, Brazil (JACoW Publishing, Geneva, 2021), https:// accelconf.web.cern.ch/ipac2021/export/FRXA02-bib.htm.

[5] C. Houghton, C. Bloomer, and L. Alianelli, J. Synchrotron Radiat. 28, 1357 (2021).

[6] A. Andersson, M. Boge, A. Ludeke, V. Schlott, and A. Streun, Nucl. Instrum. Methods Phys. Res., Sect. A 591, 437 (2008).

[7] C. Thomas, G. Rehm, I. Martin, and R. Bartolini, Phys. Rev. ST Accel. Beams 13, 022805 (2010).

[8] O. Chubar, Y.S. Chu, K. Kaznatcheev, and H. Yan, Nucl. Instrum. Methods Phys. Res., Sect. A 649, 118 (2011), National Synchrotron Radiation Instrumentation conference in 2010.

[9] O. V. Chubar, Rev. Sci. Instrum. 66, 1872 (1995).

[10] O. Chubar and P. Elleaume, Conf. Proc. C 980622, 1177 (1998), https://inspirehep.net/literature/487818.

[11] R. Bachelard, P. Mercère, M. Idir, M.-E. Couprie, M. Labat, O. Chubar, G. Lambert, P. Zeitoun, H. Kimura, H. Ohashi, A. Higashiya, M. Yabashi, M. Nagasono, T. Hara, and T. Ishikawa, Phys. Rev. Lett. 106, 234801 (2011).

[12] J. Breunlin, . Andersson, N. Milas, Sa Hernndez, and V. Schlott, Nucl. Instrum. Methods Phys. Res., Sect. A 803, 55 (2015).

[13] O. Chubar et al., https://github.com/ochubar/SRW (2013).

[14] S. Kongtawong, Y. Tian, X. Yang, K. Ha, L. H. Yu, and T. Shaftan, Nucl. Instrum. Methods Phys. Res., Sect. A 976, 164250 (2020).

[15] S. Kongtawong, Y. Tian, L. H. Yu, X. Yang, G. Wang, K. Ha, and T. Shaftan, Nucl. Instrum. Methods Phys. Res., Sect. A 997, 165175 (2021). 
[16] J. Goodman, Introduction to Fourier Optics, 4th ed. (W. H. Freeman, Macmillan Learning, San Francisco, 2017).

[17] O. Chubar, L. Berman, Y. S. Chu, A. Fluerasu, S. Hulbert, M. Idir, K. Kaznatcheev, D. Shapiro, Q. Shen, and J. Baltser, in Advances in Computational Methods for X-Ray Optics II, Vol. 8141, edited by M. S. del Rio and O. Chubar, International Society for Optics and Photonics (SPIE, 2011), pp. 44-53.

[18] A. Papoulis and S. U. Pillai, Probability, random variables, and stochastic processes, 4th ed. (McGraw-Hill, New York, 2002).
[19] L. Farvacque, in CERN Accelerator School, Synchrotron Radiation and Free Electron Lasers, edited by S. Turner (CERN, 1998), pp. 287-302.

[20] J. D. Jackson, Classical Electrodynamics, 3rd ed. (Wiley, New York, 1999).

[21] W. Cheng, B. Bacha, B. Kosciuk, and D. Padrazo, Jr., in Proceedings of 9th International Particle Accelerator Conference (IPAC'18), Vancouver, BC, Canada, April 29May 4, 2018, International Particle Accelerator Conference No. 9 (JACoW Publishing, Geneva, Switzerland, 2018), pp. 1837-1840, 10.18429/JACoW-IPAC2018-WEPAF012. 